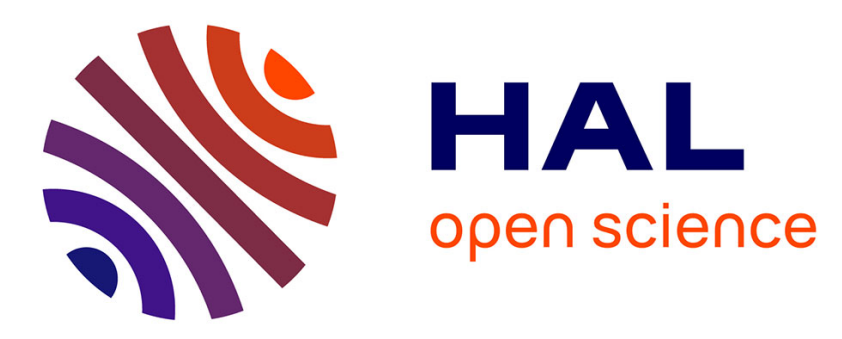

\title{
The lncRNA MARS modulates the epigenetic reprogramming of the marneral cluster in response to ABA
}

Thomas Roulé, Federico Ariel, Caroline Hartmann, Jose Gutierrez-Marcos, Nosheen Hussain, Martin Crespi, Thomas Blein

\section{To cite this version:}

Thomas Roulé, Federico Ariel, Caroline Hartmann, Jose Gutierrez-Marcos, Nosheen Hussain, et al.. The lncRNA MARS modulates the epigenetic reprogramming of the marneral cluster in response to ABA. 2020. hal-03016338

\section{HAL Id: hal-03016338 \\ https://hal.science/hal-03016338}

Preprint submitted on 20 Nov 2020

HAL is a multi-disciplinary open access archive for the deposit and dissemination of scientific research documents, whether they are published or not. The documents may come from teaching and research institutions in France or abroad, or from public or private research centers.
L'archive ouverte pluridisciplinaire HAL, est destinée au dépôt et à la diffusion de documents scientifiques de niveau recherche, publiés ou non, émanant des établissements d'enseignement et de recherche français ou étrangers, des laboratoires publics ou privés. 
1 The IncRNA MARS modulates the epigenetic reprogramming of the marneral 2 cluster in response to $\mathrm{ABA}$

3 Thomas Roulé ${ }^{1,2}$, Federico Ariel ${ }^{3}$, Caroline Hartmann ${ }^{12}$, Jose Gutierrez-Marcos ${ }^{4}$, Nosheen 4 Hussain $^{4}$, Martin Crespi ${ }^{12^{*}}$ and Thomas Blein ${ }^{12}$

Institute of Plant Sciences Paris-Saclay, Centre Nationale de la Recherche, Institut National de la Recherche Agronomique, Université Evry, Université Paris-Saclay, 91405 Orsay, France

${ }^{2}$ Institute of Plant Sciences Paris-Saclay, Université de Paris, 91405 Orsay, France

${ }^{3}$ Instituto de Agrobiotecnología del Litoral, CONICET, FBCB, Universidad Nacional del Litoral, Colectora Ruta Nacional 168 km 0, 3000 Santa Fe, Argentina

${ }^{4}$ School of Life Sciences, University of Warwick, Coventry, UK

${ }^{*}$ Correspondence to: MC (martin.crespi@universite-paris-saclay.fr)

\section{ABSTRACT}

Clustered organization of biosynthetic non-homologous genes is emerging as a characteristic feature of plant genomes. The co-regulation of clustered genes seems to largely depend on epigenetic reprogramming and three-dimensional chromatin conformation. Here we identified the long noncoding RNA (IncRNA) MARneral Silencing (MARS), localized inside the Arabidopsis marneral cluster, and which controls the local epigenetic activation of its surrounding region in response to ABA. MARS modulates the POLYCOMB REPRESSIVE COMPLEX 1 (PRC1) component LIKE-HETEROCHROMATIN PROTEIN 1 (LHP1) binding throughout the cluster in a dose-dependent manner, determining H3K27me3 deposition and chromatin condensation. In response to ABA, MARS decoys LHP1 away from the cluster and promotes the formation of a chromatin loop bringing together the MARNERAL SYNTHASE 1 (MRN1) locus and a distal ABA-responsive enhancer. The enrichment of co-regulated IncRNAs in clustered metabolic genes suggests that the acquisition of noncoding transcriptional units constitute an additional regulatory layer driving the evolution of biosynthetic pathways.

KEYWORDS: IncRNA, enhancer, cluster, chromatin conformation, LHP1, ABA, seed germination, epigenetics, marneral 


\section{INTRODUCTION}

In eukaryotes, functionally related genes are usually scattered across the genome. However, a growing number of operon-like clustered organization of non-homologous genes participating in common metabolic pathways point at an emerging feature of animal, fungi and plant genomes ${ }^{1}$.

In plants, synthesis of numerous secondary metabolic compounds is important for the dynamic interaction with their environment, affecting their life and survival ${ }^{2}$. Terpenoids are bioactive molecules of diverse chemical structure ${ }^{3}$. In Arabidopsis thaliana, the biosynthesis of four triterpenes, namely thalianol ${ }^{4}$, tirucalla-7,24-dien-3b-ol ${ }^{5}$, arabidiol ${ }^{6}$ and marneral $^{7}$, is governed by enzymes encoded by genes organized in clusters ${ }^{8}$. The thalianol and marneral related genes are located in the smallest metabolic clusters identified in plants to date, each being less than $40 \mathrm{~kb}$ in size ${ }^{8}$. Both compounds are derived from 2,3-oxidosqualene and the corresponding gene clusters contain the oxidosqualene cyclases (OSCs), thalianol synthase (THAS) and marneral synthase (MRN1), respectively. The marneral cluster includes two additional protein-coding genes, CYP705A12 and CYP71A16, participating in marneral oxidation ${ }^{7}$.

Growing evidence indicates that the co-regulation of clustered genes relies on epigenetic mechanisms. It has been shown that the deposition of the histone variant H2A.Z positively correlates with transcriptionally active clusters. Accordingly, nucleosome stability precluding gene expression is dependent on ARP6, a component of the SWR1 chromatin remodeling complex required for the deposition of $\mathrm{H} 2 \mathrm{~A} . \mathrm{Z}$ into nucleosomes ${ }^{9}$. Additionally, it was shown that the thalianol and marneral clusters exhibit increased expression in the Polycomb mutant curly leaf (clf) with compromised H3K27me3 deposition, and reduced expression in the trithorax-group protein mutant pickle (pkl), a positive regulator that counteracts $\mathrm{H} 3 \mathrm{~K} 27 \mathrm{me} 3$ silencing $^{10}$. Strikingly, it has been recently shown that biosynthetic gene clusters are embedded in local hot spots of three-dimensional (3D) contacts that segregate cluster regions from the surrounding chromosome environment in a tissuedependent manner. Notably, H3K27me3 appeared as a central feature of the 3D domains at silenced clusters ${ }^{11}$.

Long noncoding RNAs (IncRNAs) have emerged as important regulators of eukaryotic gene expression at different levels ${ }^{12}$. In plants, several IncRNAs have been shown to interact with the Polycomb Repressive Complex 1 and 2 components LIKE HETEROCHROMATIN PROTEIN 1 (LHP1) and CLF, respectively, which are related to H3K27me3 distribution ${ }^{13,14}$. Furthermore, it has been proposed that IncRNAs can modulate the transcriptional activity of 
neighboring genes by shaping local 3D chromatin conformation ${ }^{15-17}$. Here we show that the marneral cluster in Arabidopsis includes three noncoding transcriptional units. Among them, the IncRNA MARS influences the expression of marneral cluster genes in response to $A B A$ through modification of the epigenetic landscape. MARS deregulation affects H3K27me3 distribution, LHP1 deposition and chromatin condensation throughout the cluster. Furthermore, an $\mathrm{ABA}$ responsive chromatin loop dynamically regulates MRN1 transcriptional activation by bringing together the MRN1 proximal promoter and an enhancer element enriched in ABA-related transcription factors (TF) binding sites. MARS-mediated control of the marneral cluster affects seed germination in response to ABA. The general co-regulation of genes located within IncRNA-containing clusters in Arabidopsis points to noncoding transcription as an important feature in coordinated transcriptional activity of clustered loci.

\section{RESULTS}

\section{The marneral gene cluster includes three noncoding transcriptional units}

The small marneral cluster includes three genes: marneral synthase (MRN1), CYP705A12 and CYP71A16 that are two P450 cytochrome-encoding genes (Fig. 1a), all participating in the biosynthesis and metabolism of the triterpene marneral ${ }^{7}$.

The advent of novel sequencing technologies has allowed the identification of an increasing number of IncRNAs throughout the Arabidopsis genome. According to the latest annotation (Araport $11^{18}$ ), three additional transcriptional units are located within the marneral cluster, between the CYP71A16 and the MRN1 loci. The AT5G00580 and the pair of antisense genes AT5G06325 and AT5G06335 are located upstream of the MRN1 gene at $6 \mathrm{kpb}$ and 3kbp, respectively (Fig. 1a). The 1,941bp-long AT5G00580 locus generates four transcript isoforms ranging from 636 nt to 1,877 nt in length (Fig. 1b and 1c). In contrast, each of the antisense genes AT5G06325 and AT5G06335 are transcribed into only one RNA molecule of $509 \mathrm{nt}$ and $367 \mathrm{nt}$, respectively (Fig. 1a). All these transcripts were classified as IncRNAs when using two coding prediction tools, $\mathrm{CPC}^{19}$ and $\mathrm{CPC}^{20}$, because of their low coding potential and their length (over $200 \mathrm{nt}$ ), similarly to previously characterized IncRNAs (COLDAIR ${ }^{21}$; APOLO ${ }^{15}$; and $A S C O^{22}$ ) (Fig. 1d).

According to available transcriptomic datasets (Araport11), AT5G00580 transcriptional accumulation positively correlates with that of marneral genes, whereas AT5G06325 and AT5G06335 RNAs do not (Supplementary Fig. 1). Notably, our analysis of 
the transcriptional behavior of the noncoding gene AT5G00580 and the marneral cluster protein-coding genes revealed a correlated expression in response to phosphate and nitrate starvation, heat stress, as well as to exogenous auxin and ABA (Fig. 1e). Interestingly, the AT5G00580 IncRNA exhibited the strongest induction in response to heat stress and exogenous ABA, in comparison with MRN1 and the two CYP genes (Fig. 1e). Altogether, our observations uncovered that the marneral cluster includes three noncoding transcriptional units, one of which is actively transcribed and co-regulated with its neighboring proteincoding genes.

\section{The IncRNA MARS shapes the transcriptional response of the marneral gene cluster to ABA}

It has been shown that IncRNAs can regulate the expression of their neighboring genes through epigenetic mechanisms ${ }^{14,23}$. Thus, we wondered if the IncRNA derived from the AT5G00580 locus may regulate the transcriptional activity of the protein-coding genes included in the marneral cluster. To this end, we modified the IncRNA expression without affecting the cluster DNA region using an RNAi construct targeting the first exon of AT5G00580, and isolated two independent lines. The RNAi line 1 impaired the transcriptional accumulation of AT5G00580 without affecting the rest of the cluster. Interestingly, the RNAi line 2 exhibited a strong down-regulation of AT5G00580 together with a significant basal repression of the two CYP genes (Fig. 2a). Strikingly, the response of the three proteincoding genes of the marneral cluster to exogenous $A B A$ was significantly deregulated in both independent RNAi lines. Transcriptional levels of MRN1 and the two CYP genes increased earlier in RNAi seedlings (15 min) than in the wild-type (Col-0, $30 \mathrm{~min}$ ) (Fig. 2b bottom panel). In addition, the transcriptional accumulation of these genes later reached three-fold higher levels in the RNAi lines compared to Col-0 (Fig. 2b top panel). To further support our observations, we isolated the insertional mutant SALK_133089 located 200 bp upstream the transcription start site (TSS) of AT5G00580 gene. Although this insertion did not affect AT5G00580 basal levels (Fig. 2a), it partially impaired its induction in response to ABA. In agreement with both RNAi lines, MRN1 and CYP705A12 genes were strongly induced by exogenous ABA (Supplementary Fig. 2), in contrast to CYP71A16, whose promoter region may be locally affected by the T-DNA insertion. Notably, MRN1, which encodes the marneral synthase, is the gene in the cluster most strongly affected by AT5G00580 down-regulation. Collectively, our results indicate that the noncoding transcriptional activity derived from the AT5G00580 locus represses the dynamic expression of the marneral cluster genes, mainly $M R N 1$, in response to ABA. Therefore, we named the AT5G00580-derived noncoding transcript MARneral Silencing (MARS) IncRNA. 


\section{MARS affects seed germination likely through its impact on MRN1} expression

The phytohormone ABA has been implicated in the perception and transduction of environmental signals participating also in a wide range of growth and developmental events such as seed maturation, dormancy and germination ${ }^{24}$. Considering that the marneral cluster exhibited a strong MARS-dependent response to ABA, we wondered what the physiological impact of MARS deregulation was during seed germination. To this end, we assessed seed germination in Col-0 and MARS down-regulated lines (RNAi lines 1/2 and SALK_133089) with or without exogenous ABA. Notably, MARS silencing resulted in a delayed germination compared to Col-0 (Supplementary Fig. 3a), as revealed by an increase in T50 (time for $50 \%$ of germination) of nearly 5 hours (Supplementary Fig. 3b). Interestingly, in response to $0.5 \mu \mathrm{M} A B A$, the germination of RNAi-MARS was further delayed than Col-0 with an increase of T50 of nearly 10 hours (Supplementary Fig. 3c and 3d). Accordingly, transgenic plants over-expressing MRN1 also exhibited a delayed germination phenotype regardless of the treatment with ABA (Supplementary Fig. 3). The behavior of 35S:MRN1 seedlings suggests that MRN1 up-regulation in RNAi-MARS lines could be linked to the increased sensitivity to ABA (Fig. 2b). Altogether, our results indicate that MARS can modulate seed germination through the regulation of the expression of MRN1.

\section{MARS controls the epigenetic status of the marneral locus}

It has been shown that gene clusters in plants are tightly regulated by epigenetic modifications, including the repressive mark $\mathrm{H} 3 \mathrm{~K} 27 \mathrm{me} 3^{10}$. According to publicly available ChIP-Seq datasets ${ }^{25}$, the marneral cluster region is highly enriched in H3K27me3 deposition in shoots, extensively coinciding with LHP1 recognition (Supplementary Fig. 4). Consistently, an ATAC-Seq available datase ${ }^{26}$ revealed that the marneral cluster exhibits a high chromatin condensation in shoots (Supplementary Fig. 4). Thus, the marneral cluster is characterized by an epigenetically silent status in aerial organs coinciding with its low expression level ${ }^{10}$.

We wondered if the transcriptional activation of the marneral cluster in response to exogenous $A B A$ was associated with a dynamic epigenetic reprogramming. We first assessed H3K27me3 deposition across the marneral cluster, including the gene body of MRN1, MARS and the two CYP loci (Fig. 3a and Supplementary Fig. 5). Interestingly, 
exogenous $A B A$ triggered a strong reduction of $\mathrm{H} 3 \mathrm{~K} 27 \mathrm{me} 3$ deposition throughout the marneral cluster (Fig. 3a and Supplementary Fig. 5). Strikingly, H3K27me3 basal levels were also significantly lower in RNAi-MARS seedlings. Remarkably, H3K27me3 deposition was even lower across the body of all genes of the cluster in response to ABA in the RNAi$M A R S$ lines when compared with Col-0, in agreement with the stronger induction by ABA of this subset of genes upon MARS silencing (Fig. 3a, Supplementary Fig. 5 and Fig. 2b). Furthermore, we assessed LHP1 recognition of the marneral cluster. In agreement with previous observations (Supplementary Fig. $4^{25}$ ), LHP1 distribution was high in MRN1 promoter and more weakly across MARS gene body and the intergenic region between CYP71A16 and MARS (Fig. 3b and Supplementary Fig. 6). Remarkably, LHP1 recognition was strongly impaired in response to $A B A$ as well as in RNAi-MARS seedlings (Fig. $\mathbf{3 b}$ and Supplementary Fig. 6). Therefore, our results indicate that $A B A$ triggers an epigenetic reprogramming of the marneral cluster, likely in a process involving the IncRNA MARS.

\section{MARS is directly recognized by LHP1 and modulates local chromatin condensation}

It has been shown that the deposition of the repressive mark H3K27me3 and the concomitant recognition of the plant PRC1 component LHP1 are correlated with high chromatin condensation ${ }^{27}$. Therefore, we determined by Formaldehyde-Assisted Isolation of Regulatory Elements (FAIRE) the chromatin condensation of the whole marneral cluster. In contrast to Col-0 showing a highly condensed chromatin, RNAi-MARS seedlings exhibit a lower chromatin condensation in control conditions. Notably, the global chromatin status of the cluster was even less condensed in RNAi-MARS seedlings in response to ABA (Fig. 4a and Supplementary Fig. 7), in agreement with a decrease of both H3K27me3 deposition and LHP1 binding (Fig. 3b and Supplementary Fig. 6) and the concomitant transcriptional activation of the clustered genes (Fig. 2b). Consistently with our observations, Ihp1 mutant seedlings also showed a global chromatin decondensation in control conditions, comparable to Col-0 in response to ABA. Notably, further chromatin decondensation triggered by $A B A$ was completely impaired (Fig. 4b and Supplementary Fig. 8), supporting the role of LHP1 in the dynamic epigenetic silencing of the marneral cluster.

It has been shown that LHP1 can recognize RNAs in vitro ${ }^{13}$ and the IncRNA APOLO in vivo ${ }^{15}$. Moreover, it has been proposed that APOLO over-accumulation can decoy LHP1 away from target chromatin ${ }^{28}$. Therefore, we wondered whether MARS IncRNA was able to interact with the chromatin-related protein LHP1 participating in the modulation of the local epigenetic environment. Thus, we first determined that MARS was enriched in the nucleus, 
compared with total RNA, as the previously characterized IncRNAs APOLO and ASCO that interact respectively with nuclear epigenetic and splicing machineries, and the spliceosome structural ncRNA U6 (Supplementary Fig. 9a). Then, we confirmed by RNA immunoprecipitation (RIP) that LHP1 can interact with MARS in vivo, in contrast to the MRN1 mRNA or housekeeping RNAs taken as negative controls (Fig. 4c).

LHP1 binding to the marneral cluster was impaired both in response to exogenous ABA (inducing MARS, Fig. 1e and Supplementary Fig. 3b) and in RNAi-MARS seedlings, hinting at a stoichiometry-dependent action of MARS on LHP1 recognition. Therefore, we used chromatin extracts from RNAi-MARS line 1 seedling, with very low MARS transcript levels (Fig. 2a) to assess LHP1 recognition of the marneral cluster upon the addition of increasing concentrations of in vitro-transcribed MARS RNA. Strikingly, we found that low MARS RNA concentrations (between 0.01 and $0.1 \mu \mathrm{g}$ of RNA; Fig. 4d and Supplementary Fig. 9b) successfully promoted LHP1 binding to the cluster, in contrast to higher concentrations (between 1 and $10 \mu \mathrm{g}$ of RNA), supporting the relevance of MARS-LHP1 stoichiometry for LHP1-target recognition (Fig. 4d and Supplementary Fig. 9b). Altogether, our results suggest that the physical interaction of the nuclear-enriched IncRNA MARS to LHP1 modulates its binding to proximal chromatin likely participating in the modulation of the dynamic chromatin condensation of the marneral cluster.

\section{MARS expression modulates an LHP1-dependent chromatin loop} bringing together the MRN1 locus and an ABA enhancer element

It was recently observed that the spatial conformation of cluster-associated domains differs between transcriptionally active and silenced clusters. In Arabidopsis, segregating 3D contacts are distinguished among organs, in agreement with the corresponding transcriptional activity of clustered genes ${ }^{11}$. Therefore, we explored whether MARS could participate in the dynamic regulation of the local 3D chromatin conformation modulating the transcription of the marneral cluster. According to available $\mathrm{HiC}$ datasets ${ }^{25,29}$ there is a significant interaction linking the intergenic region between CYP71A16 and MARS and the MRN1 locus (indicated as "Chromatin loop" in Fig. 5a). By using Chromatin Conformation Capture $(3 \mathrm{C})$, we determined that the formation of this chromatin loop drastically increased over 30 min with exogenous ABA and remained formed for at least 4 hours (Fig 5b). Thus, the formation of this chromatin loop positively correlates with the transcriptional accumulation of the marneral cluster genes in response to ABA (Fig. 2b). 
The MARS locus is encompassed in the ABA-dependent chromatin loop (Fig. 5a). In order to determine the role of MARS in the modulation of local 3D chromatin conformation, we assessed the formation of the chromatin loop in RNAi-MARS lines. Notably, RNAi-MARS seedlings exhibit enhanced chromatin loop formation, which remained unchanged in response to exogenous ABA (Fig. 5b). Interestingly, LHP1 has been implicated in shaping local 3D conformation of target regions ${ }^{25}$, suggesting that the LHP1-MARS module may dynamically switch the epigenetic status of the marneral cluster from a condensed-linear to a decondensed-3D structured chromatin conformation. Supporting this hypothesis, Ihp1 mutant seedlings exhibited enhanced chromatin loop formation compared to Col-0 (Fig. 5c). Overall, our results demonstrate that a chromatin loop within the marneral cluster is regulated by LHP1 and the interacting IncRNA MARS, encoded in the region encompassed by the loop.

To better understand the role of the MARS-dependent chromatin loop in response to

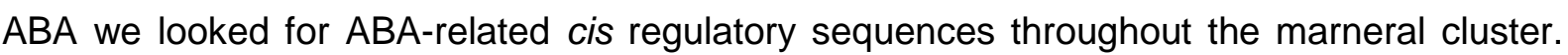
We extracted from ChIP-seq the binding distribution of 13 ABA-related transcription factors $(T F s)^{30}$. Interestingly, a high enrichment in ABA TF binding sites was found at the MARS locus, as well as in the intergenic region between the CYP71A16 and MARS loci, notably surrounding the contact point brought into close spatial proximity with the MRN1 locus by the ABA-dependent 3D chromatin loop (Fig. 5a). We thus assessed the activating capacity of this region, potentially acting as a distant enhancer element of the MRN1 proximal promoter. To this end, we made use of a GUS-based reporter system (as described in ${ }^{31}$ ), fusing two regions of interest to a minimal $35 S$ promoter. Two additional DNA regions nearby the putative enhancers were used as negative controls: one between CYP705A12 and CYP71A16 and the other at the 3' end of AT5G42620 locus (Fig. 5a indicated in red). Among the two putative enhancers regions tested, one was able to activate GUS expression (Intergenic region 2, Fig. 5a and 5d), coinciding with the region showing a high enrichment of ABA-related TF binding sites close to the chromatin loop anchor point (Fig. 5a). Collectively, our results indicate that an ABA-driven chromatin loop brings into close spatial proximity the MRN1 locus and a transcriptional activation site likely acting as an $A B A$ enhancer element. Notably, this chromatin reorganization process depends on the LHP1MARS module.

\section{Long noncoding RNAs as emerging regulators of gene clusters}

Physically linked genes organized in clusters are generally coregulated ${ }^{8}$. Considering that the IncRNA MARS is implicated in the regulation of the marneral cluster, we wondered whether the presence of noncoding transcriptional units may constitute a relevant feature of 
gene cluster organization. Therefore, we collected two lists of Arabidopsis clustered genes, i.e. one of co-expressed neighboring genes ${ }^{10}$ and one of predicted metabolic gene clusters (by PlantiSMASH ${ }^{32}$ ). According to the latest Arabidopsis genome annotation (Araport11), among the 390 clusters of co-expressed neighboring genes), 189 (48\%) contain at least one embedded IncRNA inside the cluster. Most importantly, among the 45 metabolic clusters, 28 (62\%) include IncRNAs (Fig. 6a). Furthermore, among the clusters containing a IncRNA, a correlation analysis based on the maximum strength of co-expression between a IncRNA and any clustered gene revealed that the metabolic clusters exhibit a significantly higher correlation than co-expressed clusters (Fig. 6b). Altogether, our analyses suggest that IncRNA-mediated local epigenetic remodeling may constitute an emerging feature of nonhomologous genes metabolic clusters in plants.

\section{DISCUSSION}

The cell nucleus is a dynamic arrangement of DNA, RNAs and proteins ${ }^{33,34}$. Genome topology has emerged as an important feature in the complex network of mechanisms regulating gene activity and genome connectivity, leading to regionalized chromosomal spatial distribution and the clustering of diverse genomic regions with similar expression patterns $^{35}$.

In the last few years, noncoding transcription has been implicated in shaping 3D nuclear organization ${ }^{36}$. Notably, RNase-A micro-injection into the nucleus revealed that long nuclear-retained RNAs maintained euchromatin in a biologically active decondensed state, whereas heterochromatin domains exhibited an RNA-independent structure ${ }^{37,38}$. More recently, $\mathrm{HiC}$ analyses were performed in mammalian cells exposed or not to RNase, before and after crosslinking, or upon transcriptional inhibition ${ }^{39}$. As a result, it was observed that topologically associated domains (TAD) boundaries remained mostly unaffected by RNase treatment, whereas compartmental interactions suffered a subtle disruption. In contrast, transcriptional inhibition led to weaker TAD boundaries, hinting at different roles of steadystate RNA vs. active transcription in nuclear organization ${ }^{39}$.

In plants, several IncRNAs have been implicated in local chromatin conformation dynamics affecting the transcriptional activity of neighboring genes ${ }^{14,40}$. Notably, the IncRNA COLDWRAP participates in the formation of an intragenic chromatin loop blocking the transcription of the flowering time regulator FLOWERING LOCUS $C\left(F L C^{16}\right)$ in response to PINOID (PID) by dynamically modulating the formation of an intergenic chromatin loop 
encompassing the divergent promoter of $P I D$ and $A P O L O^{15}$, in a process involving the PRC1 component LHP1. More recently, it was proposed that high levels of APOLO can decoy LHP1 away from multiple loci in trans, modulating the 3D conformation of target genes ${ }^{28}$. In rice, the expression of the leucine-rich repeat receptor kinase clustered genes $R L K s$ is modulated by the locally-encoded IncRNA LRK ANTISENSE INTERGENIC RNA (LAIR). It was proposed that $L A I R$ may directly recruit OsMOF (MALES ABSENT ON THE FIRST) and OsWDR5 (WD REPEAT DOMAIN 5), involved in H4K16 acetylation and chromatin remodeling ${ }^{41}$. Here we showed that the IncRNA MARS contributes to the co-regulation of a set of physically linked genes in cis in Arabidopsis. We demonstrated that the relative abundance of in vitro-transcribed MARS fine-tunes LHP1 binding to the cluster region in a stoichiometry-dependent manner, thus explaining how MARS affects H3K27me3 deposition and chromatin condensation. It has been shown in yeast that histone depletion boosts chromatin flexibility and facilitates chromatin loop formation on the kilobase pair scale ${ }^{42}$. In agreement thereof, we uncovered here the dynamic role of the LHP1-MARS module affecting nucleosome distribution across the marneral cluster in response to ABA, thus promoting the formation of an intra-cluster chromatin loop.

It has been recently observed that biosynthetic gene clusters are embedded in local three-dimensionally organized hot spots that segregate the region from the surrounding chromosome environment ${ }^{11}$. Here we showed that active noncoding transcriptional units within the cluster may contribute to $3 \mathrm{D}$ conformation dynamics switching from silent to active states. Our results indicated that the MARS-dependent chromatin loop may bring the MRN1 locus and a distal ABA-responsive element into close spatial proximity, likely acting as an enhancer. Notably, MARS-dependent LHP1 and H3K27me3 removal in Col-0, RNAi-MARS and the Ihp1 mutant correlated with chromatin decondensation, loop formation and increased marneral genes transcriptional activity in response to ABA. According to this model, chromatin loop conformation is related to LHP1 binding and is modulated by MARS. LHP1 recognition at basal MARS levels maintains a possibly linear conformation of the region, precluding the enhancer-MRN1 locus interaction, whereas the positively activating chromatin loop is formed in the absence of LHP1. MARS transcriptional accumulation directly modulates LHP1 binding to the marneral cluster and high levels of MARS then titrate LHP1 away from the cluster (Fig. $\mathbf{6 c}$; in response to $A B A$ ). Interestingly, when MARS levels are too low compared to basal levels (as in the RNAi lines), recruitment of LHP1 to the cluster is also impaired (Fig. 6c; MARS repression).

In mammals, growing evidence supports the role of IncRNAs in chromatin conformation determination ${ }^{43}$ and enhancer activity (e.g. $P V T 1^{44}$ and CCAT1-L ${ }^{45}$ ). Here, we 
showed that the nuclear-enriched IncRNA MARS brings together the MRN1 proximal promoter and a putative enhancer element enriched in ABA-responsive TF binding sites. Interestingly, it has been shown that human IncRNAs can modulate the binding of TFs to their target chromatin $\left(D H F R^{46}\right)$ and $P A N D A^{47}$, whereas TFs have been implicated in chromatin loop formation in plants ${ }^{35}$. Furthermore, it was shown that in addition to the TF NFYA, the IncRNA PANDA interacts with the scaffold-attachment-factor A (SAFA) as well as with PRC1 and PRC2 to modulate cell senescence ${ }^{48}$. Therefore, further research will be needed to determine what ABA-responsive TFs are in control of the marneral cluster and to elucidate how they participate in chromatin loop formation along the area, in relation with the PRC1-interacting IncRNA MARS.

Plants are a tremendous source of diverse chemicals which are important for their life and survival ${ }^{10}$. Marneral biosynthesis has been linked to root and leaf development, flowering time and embryogenesis ${ }^{2}$. Here we found that the Arabidopsis marneral cluster is activated by the phytohormone $\mathrm{ABA}$, in a IncRNA-dependent epigenetic reprogramming. MARS deregulation affects the cluster response to $A B A$, impacting seed germination. Interestingly, noncoding transcription had already been associated with seed germination. The DELAY OF GERMINATION 1 (DOG1) locus is a major actor regulating seed dormancy strength in Arabidopsis. Indeed, an antisense IncRNA (asDOG1) is able to repress DOG1 transcription in mature plants. Notably, it was observed that DOG1 suppression is released by shutting down antisense transcription, which is induced by ABA and drought ${ }^{49}$.

It was proposed that the marneral cluster was founded by the duplication of ancestral genes, independent events of gene rearrangement and the recruitment of additional genes ${ }^{7}$. The exploration of the noncoding transcriptome in Arabidopsis recently served to identify ecotype-specific IncRNA-mediated responses to the environment ${ }^{50}$. It was suggested that the noncoding genome may participate in multiple mechanisms involved in ecotype adaptation. Collectively, our results indicate that the acquisition of novel noncoding transcriptional units within biosynthetic gene clusters may constitute an additional regulatory layer behind their natural variation in plant evolution.

\section{METHODS}

\section{Lead contact and materials availability}


Further information and requests for resources and reagents should be directed to

Plant lines generated in this study are available from the Lead Contact with a completed Materials Transfer Agreement.

\section{Lines selection and generation}

All plants used in this study are in Columbia-0 background. RNAi-MARS were obtained using the pFRN binary vector ${ }^{51}$ bearing $250 \mathrm{bp}$ of the first exon of MARS gene (see primers in Supplementary Table 1), previously sub-cloned into the pENTR/D-TOPO vector. Arabidopsis plants were transformed using Agrobacterium tumefaciens Agl- $0^{52}$. The T-DNA inserted line SALK_133089 was ordered to NASC (N633089).

\section{Growth conditions}

Seeds were sown in plates vertically disposed in a growing chamber in long day conditions (16 $\mathrm{h}$ in light $150 \mathrm{uE} ; 8 \mathrm{~h}$ in dark; $21^{\circ} \mathrm{C}$ ) for all the experiments. Plants were grown on solid half-strength MS medium (MS/2) supplemented with $0.7 \%$ sucrose, and without sucrose for the germination assay. For nitrate starvation assay, $\mathrm{KNO}_{3}$ and $\mathrm{Ca}\left(\mathrm{NO}_{3}\right)_{2}$ were replaced from $\mathrm{MS} / 2$ by a corresponding amount of $\mathrm{KCl}$ and $\mathrm{CaCl}_{2}$ respectively, $2.25 \mathrm{mM}$ $\mathrm{NH}_{4} \mathrm{HCO}_{3}$ was added for nitrate-containing medium. For the phosphate starvation assay, growth medium contained $0.15 \mathrm{mM} \mathrm{MgSO}_{4}, 2.1 \mathrm{mM} \mathrm{NH}_{4} \mathrm{NO}_{3}, 1.9 \mathrm{mM} \mathrm{KNO}_{3}, 0.34 \mathrm{mM} \mathrm{CaCl}_{2}$, $0.5 \mu \mathrm{M} \mathrm{KI}, 10 \mu \mathrm{M} \mathrm{FeCl}_{2}, 10 \mu \mathrm{M} \mathrm{H}_{3} \mathrm{BO}_{3}, 10 \mu \mathrm{M} \mathrm{MnSO}_{4}, 3 \mu \mathrm{M} \mathrm{ZnSO}_{4}, 0.1 \mu \mathrm{M} \mathrm{CuSO}, 0.1 \mu \mathrm{M}$ $\mathrm{CoCl}_{2}, 0.1 \mu \mathrm{M} \mathrm{Na}_{2} \mathrm{MoO}_{4}, 0.5 \mathrm{~g} \cdot \mathrm{L}^{-1}$ sucrose supplemented with $500 \mathrm{uM} \mathrm{Pi}$ for Pi containing medium versus $10 \mathrm{uM}$ for $\mathrm{Pi}$ free medium. All media were supplemented with $0.8 \mathrm{~g} / \mathrm{L}$ agar (Sigma-Aldrich, A1296 \#BCBL6182V) and buffered at pH 5.6 with 3.4mM 2-(N-morpholino) ethane sulfonic acid. For the treatment with exogenous $A B A$ or auxin, seedlings were sprayed with 10uM ABA and 10uM 1-Naphthaleneacetic acid (NAA), respectively. For heat stress, plates were transferred to a growth chamber at $37^{\circ} \mathrm{C}$ under the same lightning conditions. For nitrate and phosphate starvation assays, seedlings have been transferred at day 12 after sowing (DAS) from respectively nitrate and phosphate containing medium to nitrate and phosphate free medium. Finally, for seed germination assay, 0.5uM ABA was supplemented or not to the medium. Germination rate was evaluated twice a day. Seeds were considered germinated when the seed coats were perforated by elongating radicle. For all the experiments, samples were taken from 12 DAS starting two hours after light illumination, at different time-points, after cross-linking or not, depending on the experiment. 
Total RNA was extracted from whole seedlings using TRI Reagent (Sigam-Aldrich) and treated with DNase (Fermentas) as indicated by the manufacturers. Reverse transcription was realized on lug total RNA using the Maxima Reverse Transcriptase (Thermo Scientific). qPCR was performed on a Light Cycler 480 with SYBR Green master I

400

401

402

403 (Roche) in standard protocol (40 cycles, $60^{\circ} \mathrm{C}$ annealing). Primers used in this study are listed in Supplementary Table 1. Data were analyzed using the delta delta $\mathrm{Ct}$ method using PROTEIN PHOSPHATASE 2A SUBUNIT A3 (AT1G13320) for gene normalization ${ }^{53}$ and time 0 for time-course experiment.

\section{Chromatin Immunoprecipitation (ChIP)}

ChIP was performed using anti-lgG (Millipore,Cat\#12-370), anti-H3K27me3 (Millipore, Cat\#07-449) and anti-LHP1 (Covalab, Pab0923-P), as previously described ${ }^{15}$, starting from two grams of seedlings crosslinked in $1 \%(\mathrm{v} / \mathrm{v})$ formaldehyde. Chromatin was sonicated in a water bath Bioruptor Plus (Diagenode; 60 cycles of 30s ON and 30s OFF pulses at high intensity). ChIP was performed in an SX-8G IP-Star Compact Automated System (Diagenode). Antibody coated into Protein A Dynabeads (Invitrogen) were incubated 12 hours at $4{ }^{\circ} \mathrm{C}$ with the samples. Recovering of immunoprecipitated DNA was realized using Phenol:Chloroform:Isoamilic Acid (25:24:1, Sigma) followed by ethanol precipitation and analyzed by qPCR. For input samples, non-immunoprecipitated sonicated chromatin was processed in parallel.

In-vitro transcribed MARS RNA was obtained from a PCR product amplified from wildtype genomic DNA using the T7 promoter on the 5' PCR primer (Supplementary Table 1). PCR products were verified using agarose electrophoresis, and purified using NucleoSpin kit (Macherey-Nagel). $1 \mu \mathrm{g}$ of purified DNA was used for in-vitro transcription following the manufacturer instructions (HiScribe T7 High Yield RNA Synthesis Kit, NEB). Purified noncrosslinked chromatin obtained from five grams of MARS RNAi line 1 seedlings were resuspending in $1 \mathrm{~mL}$ of nuclei lysis buffer and split into five tubes. An increasing quantity of MARS RNA was added to each tube from 0 to $10 \mu \mathrm{g}$ RNA and incubated under soft rotation during $3 \mathrm{~h}$ at $4{ }^{\circ} \mathrm{C}$. Chromatin samples were then cross-linked using $1 \%(\mathrm{v} / \mathrm{v})$ of formaldehyde for five minutes. Sonication and the following ChIP steps were performed as above.

\section{Formaldehyde-Assisted Isolation of Regulatory Elements (FAIRE)}


FAIRE was performed as described by ${ }^{54}$. After chromatin purification as for ChIP, only $50 \mu \mathrm{l}$ from the $500 \mu \mathrm{l}$ of purified chromatin were used (diluted to $500 \mu \mathrm{l}$ with $10 \mathrm{mM}$ Tris$\mathrm{HCl} \mathrm{pH}$ 8). For the qPCR, the same set of primers as for ChIP were used.

\section{Nuclear purification}

Non-cross-linked seedlings were used to assess the sub-cellular localization of RNAs. To obtain the nuclear fraction, chromatin was purified as for ChIP and resuspended, after the sucrose gradient, into $1 \mathrm{~mL}$ of TRI Reagent (Sigam-Aldrich). For the total fraction, $200 \mu \mathrm{L}$ of cell suspension in cell resuspension solution, were collected and completed with $800 \mu \mathrm{L}$ of TRI Reagent to follow with the RNA extraction. RNA's samples were treated by DNase, and $\mathrm{RT}$ was performed using random hexamers prior to qPCR analysis.

\section{RNA immunoprecipitation (RIP)}

For RIP, the Ihp1 mutant complemented with the ProLHP1:LHP1:GFP 55 were used after 4 hours of treatment with ABA. After crosslinking and chromatin extraction as for ChIP, ten percent of resuspended chromatin was conserved at $-20^{\circ} \mathrm{C}$ as the input. Chromatin was sonicated in a water bath Bioruptor Plus (Diagenode; 5 cycles of $30 \mathrm{~s}$ ON and $30 \mathrm{~s}$ OFF pulses at high intensity). Anti-LHP1 RIP was performed using the anti-GFP antibody (Abcam ab290), as previously described ${ }^{15}$. Results were expressed as the percentage of cDNA detected after IP taking the input value as $100 \%$.

\section{Chromosome conformation capture (3C)}

$3 \mathrm{C}$ was performed as described by ${ }^{56}$. Briefly, chromatin was extracted from two grams of cross-linked seedlings as for ChIP. Overnight digestion at $37^{\circ} \mathrm{C}$ were performed using $400 \mathrm{U}$ of Hind III enzyme (NEB). Digested DNA was ligated during $5 \mathrm{~h}$ incubation at 16 ${ }^{\circ} \mathrm{C}$ with $100 \mathrm{U}$ of T4 DNA ligase (NEB). DNA was recovered after reverse crosslinking and Proteinase $\mathrm{K}$ treatment (Invitrogen) by Phenol:Chloroform:Isoamyl Acid (25:24:1; Sigma) extraction and ethanol precipitation. Interaction frequency was calculated by qPCR using a DNA region uncut by Hind III to normalize the amount of DNA.

\section{Transcriptional activation assay in tobacco leaves}

The GUS reporter system for validating the activity of the putative enhancer element was adapted from ${ }^{31}$. Different DNA fragments were cloned in the GreenGate system ${ }^{57}$ fused to a minimal 35S promoter element from CAMV (synthesized by Eurofins Genomics). The 
sub-unit B3 from 35S promoter element from CAMV ${ }^{58}$ was synthesized and used as a positive control. All primers used for cloning are indicated in Supplementary Table 1.

A. tumefaciens-mediated transient transformation was performed on 5-week-old tobacco plants using a needle-less syringe. Together with enhancer constructs, another vector containing mCherry driven by $35 \mathrm{~S}$ promoter was co-transfected to control the transformation efficiency. Two leaf discs were collected near the infiltration site. One, to determine the transfection efficiency by mCherry fluorescence observation under epifluorescent microscope. The second was used for GUS staining, as previously described $^{59}$. Samples were incubated $4 \mathrm{~h}$ in the dark at $37^{\circ} \mathrm{C}$ before observation.

\section{Identification of IncRNA loci in Arabidopsis gene clusters}

The genes of co-expressed clusters were retrieved from ${ }^{10}$. The boundaries of the gene clusters were extracted using Araport11 annotations. The boundaries of the metabolic clusters were extracted from the plantiSMASH predicted clusters on Arabidopsis ${ }^{32}$. Using Araport11 GFF, the IncRNAs (genes with a locus type annotated as "long_noncoding_rna", "novel_transcribed_region" or "other_rna") present within the boundaries of the cluster were retrieved.

\section{Gene expression correlation analysis}

To compute the correlation of expression in different organ of Arabidopsis we used the 113 RNA-seq datasets that were used for the Araport11 annotations (10.1111/tpj.13415). These datasets were generated from untreated or mock-treated wild-type Col-0 plants. After removing the adaptors with Trim Galore with default parameters, the reads were mapped on TAIR10 with STAR v2.7.2a (10.1093/bioinformatics/bts635) and the parameters '-alignIntronMin 20 --alignIntronMax 3000'. Gene expression was then quantified with featureCounts v2.0.0 (10.1093/bioinformatics/btt656) with the parameters "-B -C -p -s 0" using the GFF of Araport11. Raw counts were then normalized by median of ratios using the DESeq2 R package ${ }^{60}$.

For the correlation of expression inside the marneral cluster, the expression of the genes of the clusters and 25kb around it (four genes upstream and two downstream) were collected for the correlation analysis. Pearson's correlations for each pair of genes were computed after log 2 transformation of the normalized counts. The correlation value and associated $p$-value were plotted with the corrplot $R$ package ${ }^{61}$. 
Inside each co-expressed and metabolic clusters of genes, Pearson's correlation was computed between every possible pairs of IncRNA and coding gene as for the genes inside the marneral cluster. The maximum correlation value was kept as an indication of IncRNAs correlation with the genes of the cluster.

\section{Quantification and statistical analyses}

For all the experiments, at least two independent biological samples were considered. For RT-qPCR, each sample was prepared from a pool of 5 to 10 individual seedlings. For biochemistry assays (ChIP, FAIRE, nuclear purification, RIP and 3 C) two to five grams of seedlings were prepared for each independent biological sample. For validation of enhancer function, the four leaf discs were taken from four independent tobacco plants. The tests used for statistical analyses are indicated in the respective figure legends. Statistical test and associated plots have been generated using $R$ software $\left(v 3.6 .3^{62}\right)$ with the help of the tidyverse package ${ }^{63}$.

\section{ACKNOWLEDGMENTS}

IPS2 benefits from the support of Saclay Plant Sciences-SPS (ANR-17-EUR-0007). We thank Jeremie Bazin and Aurélie Christ from IPS2 for the helpful discussion about results interpretation and design of the experiments. We also thank Moussa Benhamed (IPS2) for helpful advice on epigenetic regulation. We thank Olivier Martin for critical reading of the manuscript.

\section{AUTHORS' CONTRIBUTIONS}

TR, FA, TB and MC conceived and designed the experiments. TR performed the experiments. TR and TB analyzed the data. All authors discussed the results and edited the manuscript. 
Rev. Genet. 52, 159-183 (2018).

516

517

518

2. Go, Y. S. et al. Identification of marneral synthase, which is critical for growth and development in Arabidopsis. Plant J. 72, 791-804 (2012).

3. Yasumoto, S., Fukushima, E. O., Seki, H. \& Muranaka, T. Novel triterpene oxidizing activity of Arabidopsis thaliana CYP716A subfamily enzymes. FEBS Lett. 590, 533-540 (2016).

4. Field, B. \& Osbourn, A. E. Clusters in Different Plants. Science (80-. ). 194, 543-547 (2008).

5. Boutanaev, A. M. et al. Investigation of terpene diversification across multiple sequenced plant genomes. Proc. Natl. Acad. Sci. U. S. A. 112, E81-E88 (2015).

6. Castillo, D. A., Kolesnikova, M. D. \& Matsuda, S. P. T. An effective strategy for exploring unknown metabolic pathways by genome mining. J. Am. Chem. Soc. 135, 5885-5894 (2013).

7. Field, B. et al. Formation of plant metabolic gene clusters within dynamic chromosomal regions. Proc. Natl. Acad. Sci. 108, 16116-16121 (2011).

8. Nützmann, H. W., Huang, A. \& Osbourn, A. Plant metabolic clusters - from genetics to genomics. New Phytol. 211, 771-789 (2016).

9. Nützmann, H. W. \& Osbourn, A. Regulation of metabolic gene clusters in Arabidopsis thaliana. New Phytol. 205, 503-510 (2015).

10. Yu, N. et al. Delineation of metabolic gene clusters in plant genomes by chromatin signatures. Nucleic Acids Res. 44, 2255-2265 (2016).

11. Nützmann, H. et al. Active and repressed biosynthetic gene clusters have spatially distinct chromosome states. 1-10 (2020) doi:10.1073/pnas.1920474117.

12. Rinn, J. L. \& Chang, H. Y. Long Noncoding RNAs: Molecular Modalities to Organismal Functions. Annu. Rev. Biochem. 89, 283-308 (2020).

13. Berry, S., Rosa, S., Howard, M., Bühler, M. \& Dean, C. Disruption of an RNA-binding hinge region abolishes LHP1-mediated epigenetic repression. Genes Dev. 31, 2115-2120 (2017).

14. Lucero, L., Fonouni-Farde, C., Crespi, M. \& Ariel, F. Long noncoding RNAs shape transcription in plants. Transcription 00, 1-12 (2020).

15. Ariel, F. et al. Noncoding transcription by alternative rna polymerases dynamically regulates an auxin-driven chromatin loop. Mol. Cell 55, 383-396 (2014).

16. Kim, D.-H. \& Sung, S. Vernalization-triggered intragenic chromatin-loop formation by long 
noncoding RNAs. Dev. Cell 176, 100-106 (2017).

17. Gagliardi, D. et al. Dynamic regulation of chromatin topology and transcription by inverted repeat-derived small RNAs in sunflower. Proc. Natl. Acad. Sci. U. S. A. 116, 17578-17583 (2019).

18. Cheng, C. Y. et al. Araport11: a complete reannotation of the Arabidopsis thaliana reference genome. Plant J. 89, 789-804 (2017).

19. Kong, L. et al. CPC: Assess the protein-coding potential of transcripts using sequence features and support vector machine. Nucleic Acids Res. 35, 345-349 (2007).

552

20. Kang, Y. J. et al. CPC2: A fast and accurate coding potential calculator based on sequence intrinsic features. Nucleic Acids Res. 45, W12-W16 (2017).

21. Heo, J. B. \& Sung, S. Vernalization-mediated epigenetic silencing by a long intronic noncoding RNA. Science (80-. ). 331, 76-79 (2011).

22. Bardou, F. et al. Long Noncoding RNA Modulates Alternative Splicing Regulators in Arabidopsis. Dev. Cell 30, 166-176 (2014).

23. Jarroux, J., Morillon, A. \& Pinskaya, M. Long Non Coding RNA Biology. Adv. Exp. Med. Biol. 1008, 1-46 (2017).

24. Vishwakarma, K. et al. Abscisic acid signaling and abiotic stress tolerance in plants: A review on current knowledge and future prospects. Front. Plant Sci. 8, 1-12 (2017).

25. Veluchamy, A. et al. LHP1 Regulates H3K27me3 Spreading and Shapes the Three-Dimensional Conformation of the Arabidopsis Genome. PLoS One 11, 1-25 (2016). accessibility between Arabidopsis stem cells and mesophyll cells illuminate cell type-specific transcription factor networks. Plant J. 94, 215-231 (2018).

27. Yang, X., Tong, A., Yan, B. \& Wang, X. Governing the silencing state of chromatin: The roles of polycomb repressive complex 1 in arabidopsis. Plant Cell Physiol. 58, 198-206 (2017).

28. Ariel, F. et al. R-Loop Mediated trans Action of the APOLO Long Noncoding RNA. Mol. Cell 77, $1-11(2020)$. 
30. Song, L. et al. A transcription factor hierarchy defines an environmental stress response network. Science (80-. ). 354, 598 (2016).

31. Yan, W. et al. Dynamic control of enhancer activity drives stage-specific gene expression during flower morphogenesis. Nat. Commun. 10, 1-16 (2019).

32. Kautsar, S. A., Suarez Duran, H. G., Blin, K., Osbourn, A. \& Medema, M. H. PlantiSMASH: Automated identification, annotation and expression analysis of plant biosynthetic gene clusters. Nucleic Acids Res. 45, W55-W63 (2017).

33. Cavalli, G. \& Misteli, T. Functional implications of genome topology. Nat. Struct. Mol. Biol. 20, 290-299 (2013).

34. Gibcus, J. H. \& Dekker, J. The Hierarchy of the 3D Genome. Mol. Cell 49, 773-782 (2013).

35. Rodriguez-Granados, N. Y. et al. Put your 3D glasses on: Plant chromatin is on show. J. Exp. Bot. 67, 3205-3221 (2016).

36. Quinodoz, S. \& Guttman, M. Long non-coding RNAs: An emerging link between gene regulation and nuclear organization. Trends Cell Biol. 24, 651-663 (2014).

37. Caudron-Herger, M. et al. Coding RNAs with a non-coding function: Maintenance of open chromatin structure. Nucleus 2, (2011).

38. Caudron-Herger, M. \& Rippe, K. Nuclear architecture by RNA. Curr. Opin. Genet. Dev. 22, 179187 (2012).

39. Barutcu, A. R., Blencowe, B. J. \& Rinn, J. L. Differential contribution of steady-state RNA and active transcription in chromatin organization . EMBO Rep. 20, 1-13 (2019).

40. Gagliardi, D. \& Manavella, P. A. Short-range regulatory chromatin loops in plants. New Phytol. 1-6 (2020) doi:10.1111/nph.16632.

41. Wang, Y. et al. Overexpressing IncRNA LAIR increases grain yield and regulates neighbouring gene cluster expression in rice. Nat. Commun. 9, 1-9 (2018).

42. Diesinger, P. M., Kunkel, S., Langowski, J. \& Heermann, D. W. Histone depletion facilitates chromatin loops on the kilobasepair scale. Biophys. J. 99, 2995-3001 (2010).

43. Gil, N. \& Ulitsky, I. Regulation of gene expression by cis-acting long non-coding RNAs. Nat. Rev. Genet. 21, 102-117 (2020). 
45. Xiang, J. F. et al. Human colorectal cancer-specific CCAT1-L IncRNA regulates long-range chromatin interactions at the MYC locus. Cell Res. 24, 513-531 (2014).

605

606

607

608

609

610

611

46. Martianov, I., Ramadass, A., Serra Barros, A., Chow, N. \& Akoulitchev, A. Repression of the human dihydrofolate reductase gene by a non-coding interfering transcript. Nature $\mathbf{4 4 5}, 666-$ 670 (2007).

47. Hung, T. et al. Extensive and coordinated transcription of noncoding RNAs within cell-cycle promoters. Nat. Genet. 43, 621-629 (2011).

48. Puvvula, P. K. et al. Long noncoding RNA PANDA and scaffold-attachment-factor SAFA control senescence entry and exit. Nat. Commun. 5, (2014).

49. Yatusevich, R. et al. Antisense transcription represses Arabidopsis seed dormancy QTL DOG 1 to regulate drought tolerance . EMBO Rep. 18, 2186-2196 (2017).

50. Blein, T. et al. Landscape of the non-coding transcriptome response of two Arabidopsis ecotypes to phosphate starvation. Plant Physiol. 183, pp.00446.2020 (2020).

51. Ariel, F. et al. Two direct targets of cytokinin signaling regulate symbiotic nodulation in medicago truncatula. Plant Cell 24, 3838-3852 (2012).

52. Clough, S. J. \& Bent, A. F. Floral dip: A simplified method for Agrobacterium-mediated transformation of Arabidopsis thaliana. Plant J. 16, 735-743 (1998).

53. Czechowski, T., Stitt, M., Altmann, T., Udvardi, M. K. \& Scheible, W. Genome-Wide Identification and Testing of Superior Reference Genes for Transcript Normalization in Arabidopsis. Plant Physiol. 139, 5-17 (2005).

54. Simon, J. M., Giresi, P. G., Davis, I. J. \& Lieb, J. D. Using formaldehyde-assisted isolation of regulatory elements (FAIRE) to isolate active regulatory DNA. Nat. Protoc. 7, 256-267 (2012).

55. Nakahigashi, K., Jasencakova, Z., Schubert, I. \& Goto, K. The Arabidopsis HETEROCHROMATIN PROTEIN1 homolog (TERMINAL FLOWER2) silences genes within the euchromatic region but not genes positioned in heterochromatin. Plant Cell Physiol. 46, 1747-1756 (2005).

56. Louwers, M., Splinter, E., van Driel, R., de Laat, W. \& Stam, M. Studying physical chromatin interactions in plants using Chromosome Conformation Capture (3C). Nat. Protoc. 4, 12161229 (2009). 
631 57. Lampropoulos, A. et al. GreenGate - A novel, versatile, and efficient cloning system for plant transgenesis. PLoS One 8, (2013).

633 58. Moreno-risueno, M. A. et al. NIH Public Access. 329, 1306-1311 (2010).

634 59. Jefferson, R. A., Kavanagh, T. A. \& Bevan, M. W. GUS fusions: ,B-glucuronidase as a sensitive and versatile gene fusion marker in higher plants. EMBO J. 6, 3901-3907 (1987).

60. Love, M. I., Huber, W. \& Anders, S. Moderated estimation of fold change and dispersion for RNA-seq data with DESeq2. Genome Biol. 15, 1-21 (2014).

638 61. Taiyun Wei and Viliam Simko (2017). R package "corrplot": Visualization of a Correlation 639 Matrix (Version 0.84). Available online at https://github.com/taiyun/corrplot

640 62. R Core Team (2018). R: A language and environment for statistical computing. R Foundation 641 for Statistical Computing, Vienna, Austria. Available online at https://www.R-project.org/.

642 63. Wickham, H. et al. Welcome to the Tidyverse. J. Open Source Softw. 4, 1686 (2019). 
GioRxiv preprint doi: https

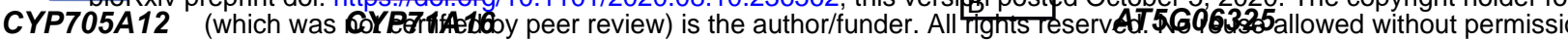

b

\section{AT5G00580}

AT5G00580.1

AT5G00580.2

AT5G00580.3

AT5G00580.4

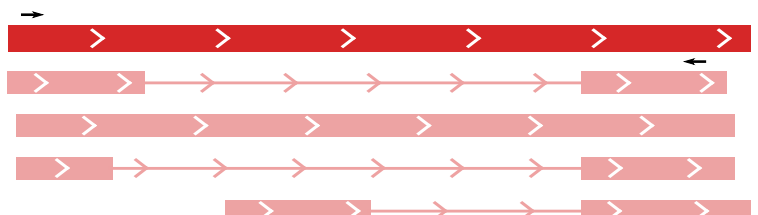

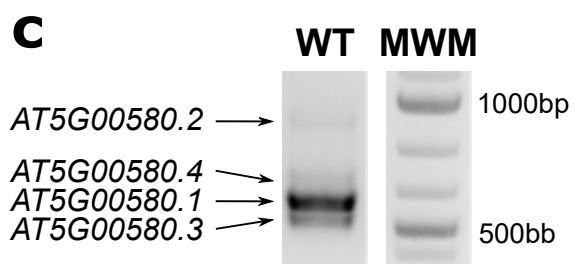

d

CPC1

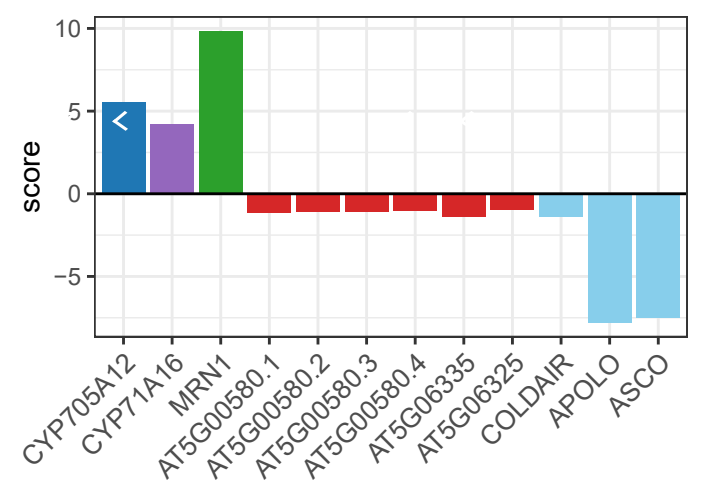

\section{CPC2}

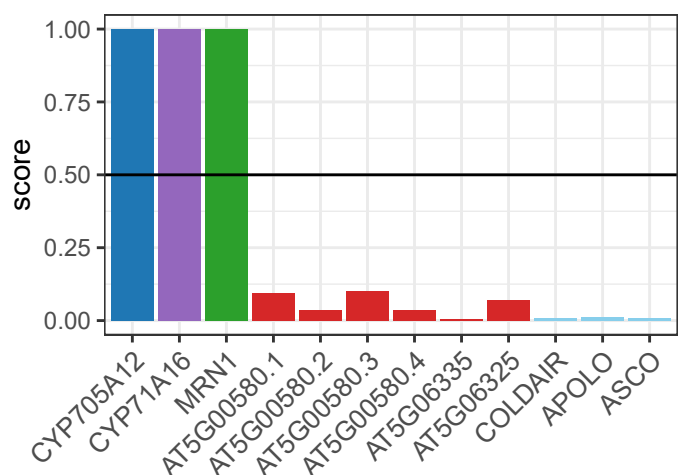

e Transcript abundance
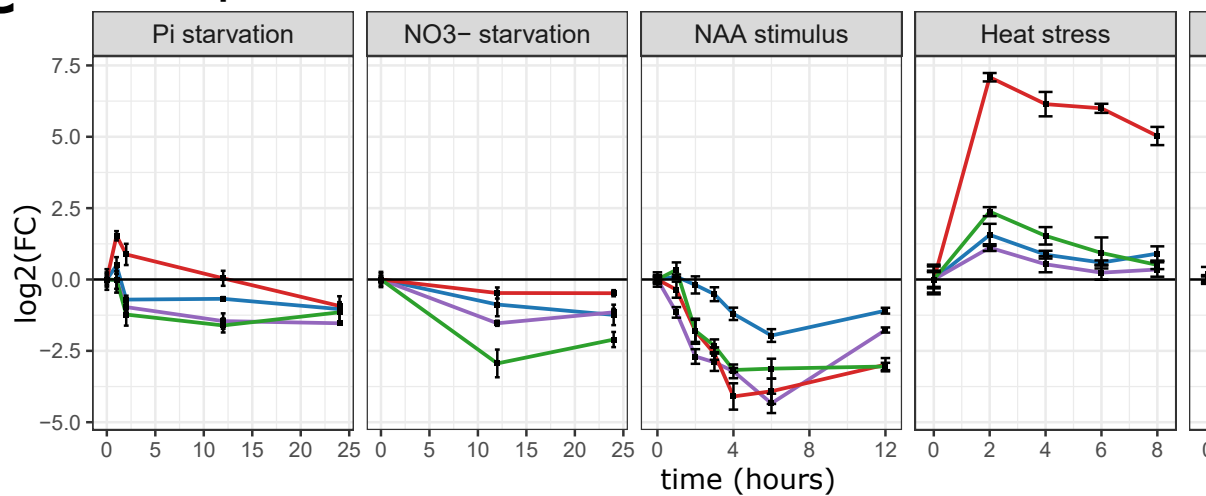

ABA stimulus

Fig. 1, AT5G00580 is a IncRNA transcribed from the marneral cluster locus and its expression correlates with its neighboring genes

a, Schematic illustration of the marneral cluster. Genes are indicated with plain rectangles and white arrows indicate the sense of transcription. The square indicates the region displayed in (B).

b, Schematic illustration of the different isoforms of $A T 5 G 00580$ transcripts. First line corresponds to AT5G00580 genomic region whereas the other lines present the various isoforms. For each isoform, exons are indicated with rectangles and introns with solid lines. Black arrows indicate the primers used for the cDNA amplification in (C).

c, cDNA amplification of AT5G00580 isoforms. The position of the primers used for the amplification are displayed on B. MWM stands for Molecular Weight Marker (GeneRuler $1 \mathrm{~kb}$ Plus DNA Ladder, Thermo Scientific).

d, Coding potential of the transcripts located in the marneral cluster genomic region. Scores were calculated using CPC1 (left) and CPC2 (right) programs ${ }^{19,20}$. For each algorithm, the threshold between coding and non-coding genes is displayed with a horizontal solid black line. Coding genes are situated above the threshold, whereas non coding genes are situated below. COLDAIR, APOLO and ASCO are used as positive controls of non-coding transcripts.

e, Dynamic transcriptional levels of co-regulated genes of the marneral cluster in response to phosphate and nitrate starvation, heat stress, and exogenous ABA and auxin. Gene expression data are represented as the mean \pm standard error $(n \geq 3)$ of the log2 fold change compared to time $0 \mathrm{~h}$. 
a Transcript abundance

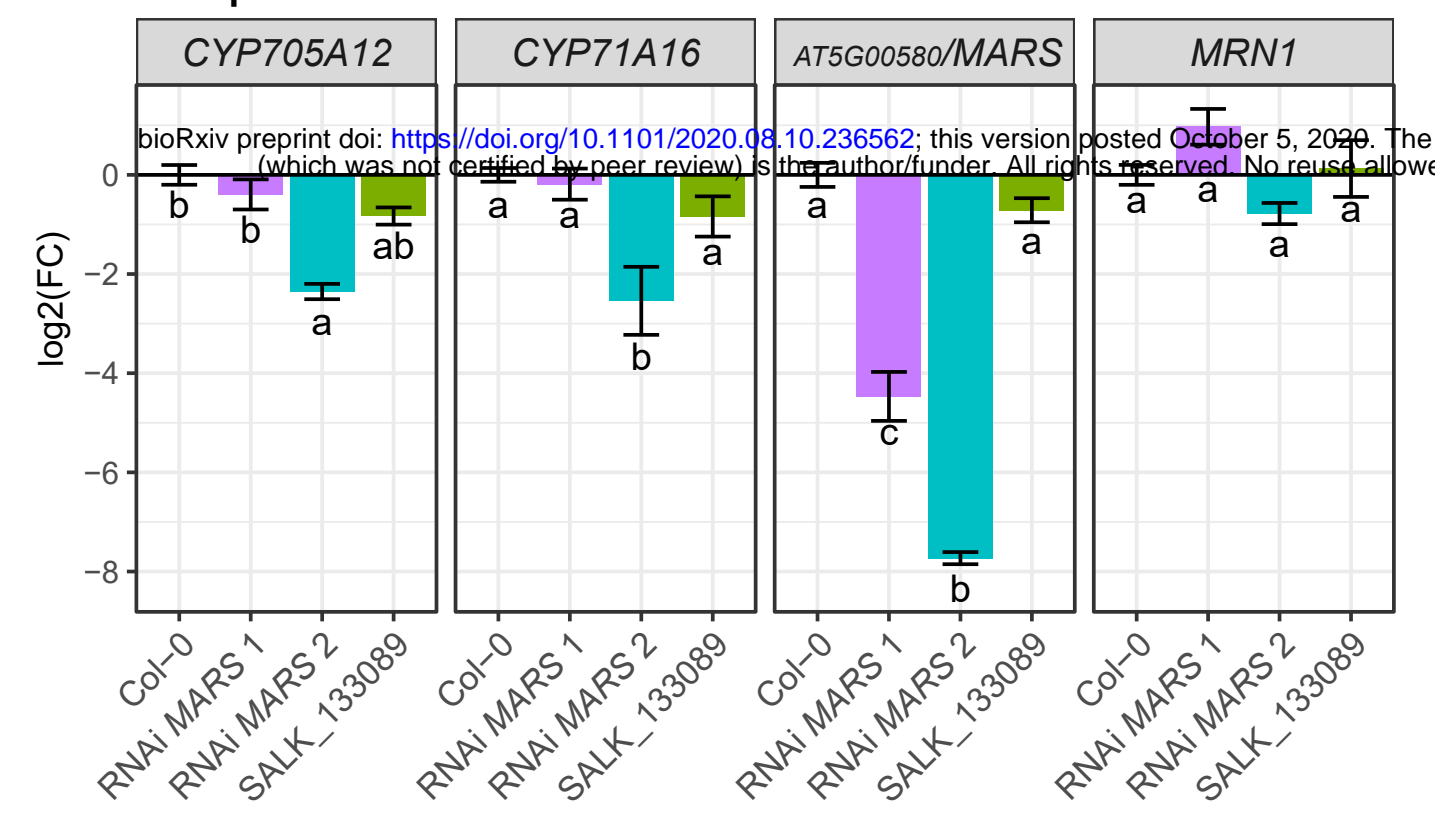

b Transcript abundance
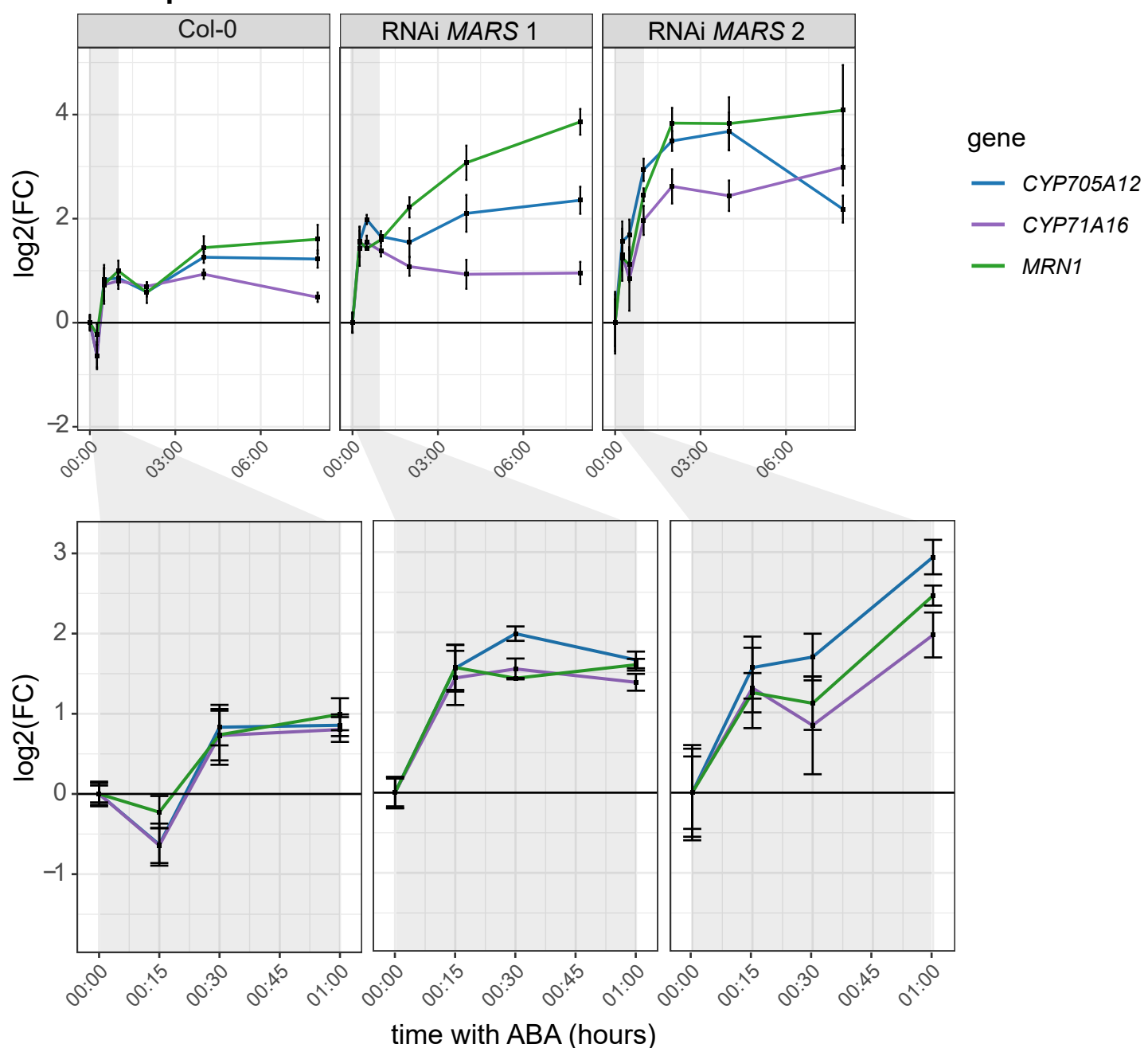
Fig. 2, MARS transcriptional activity modulates the response to ABA of the
marneral cluster

a, Transcript levels of the marneral cluster genes in control conditions in RNAi lines targeting AT5G00580/MARS and SALK_133089 line. Transcriptional levels are represented as the mean \pm standard error $(n=3)$ of the log2 fold change compared to Col-0. Letters indicate statistic group determined by one-way analysis of variance (ANOVA) followed by Tukey's post-hoc test. For each gene, each letter indicates statistical difference between genotypes $(p \leq 0.05)$.

b, Transcript levels of the genes of the marneral cluster in response to ABA treatment in RNAi lines targeting AT5G00580/MARS. Gene expression data are represented as the mean \pm standard error $(n=3)$ of the log2 fold change compared to time $0 \mathrm{~h}$. 
a H3K27me3

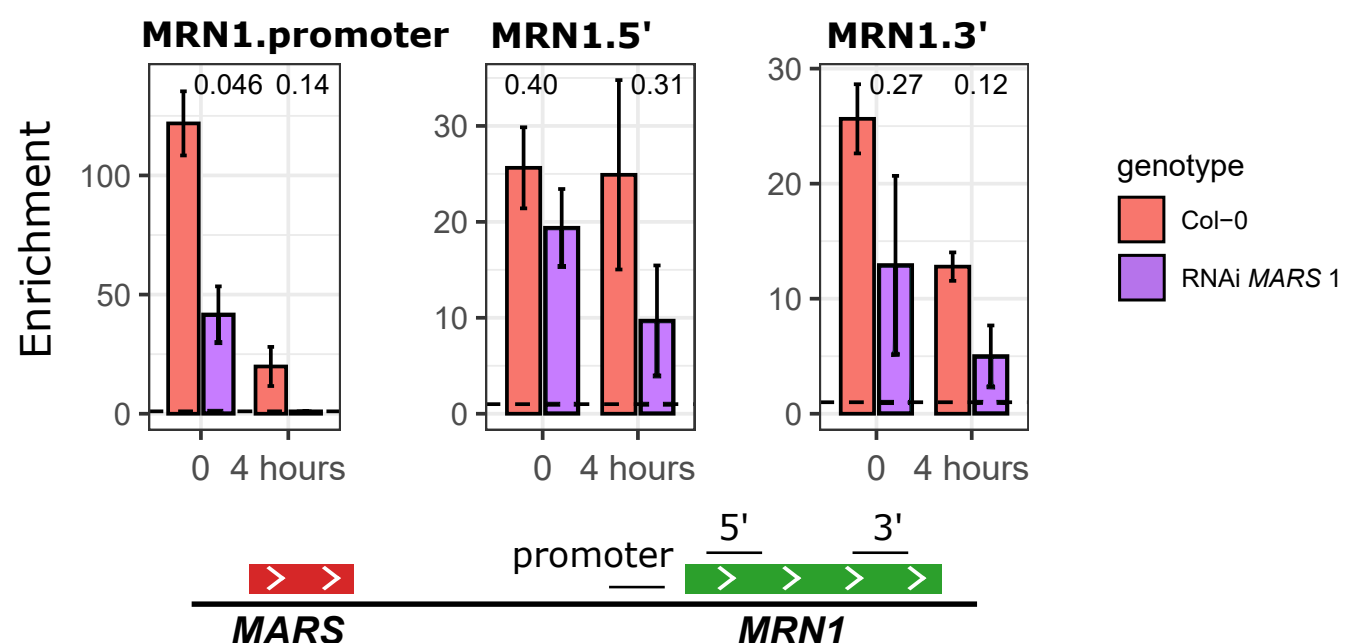

b LHP1
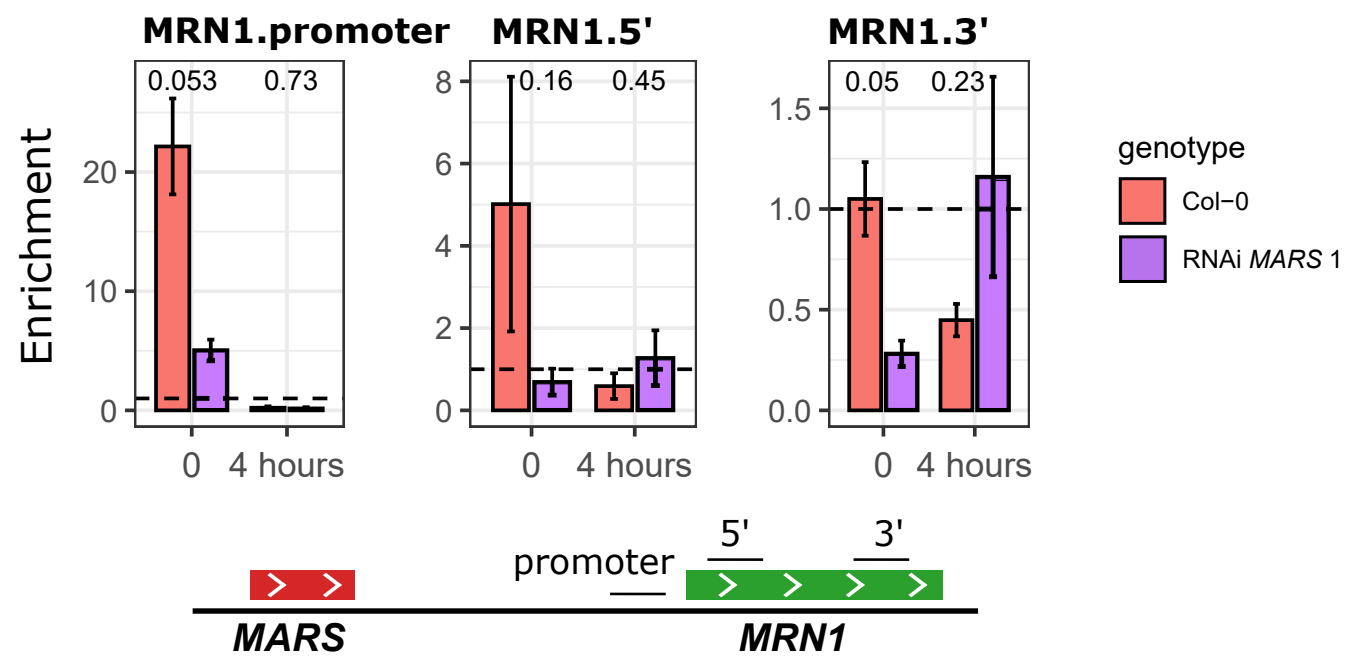

Fig. 3, MARS modulates the epigenetic landscape of MRN1 locus

a, H3K27me3 deposition over the MRN1 gene in Col-0 and RNAi-MARS seedlings under control conditions and in response to ABA. Higher values of ChIP-qPCR indicate more H3K27me3.

b, LHP1 binding to the MRN1 gene in Col-0 and RNAi-MARS seedlings in the same conditions as in (A). Higher values of ChIP-qPCR indicate more LHP1 deposition.In (A) and $(B)$, values under the dotted line are considered as not enriched. Results are represented as the mean \pm standard error $(n=2)$ of the H3K27me3/lgG or LHP1/lgG ratio. Numbers are $p$ value of the difference between the two genotypes determined by Student t-test. 
04 hours

04 hours

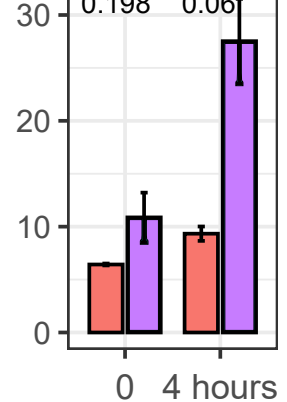

genotype

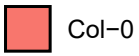

$\square$ RNAi MARS 1

bioRxiv preprint doi: https://doi.org/1prametaror $5^{\prime}, 3^{\prime}$

MARS

$3^{\prime}$

\section{MRN1}

\section{b FAIRE}

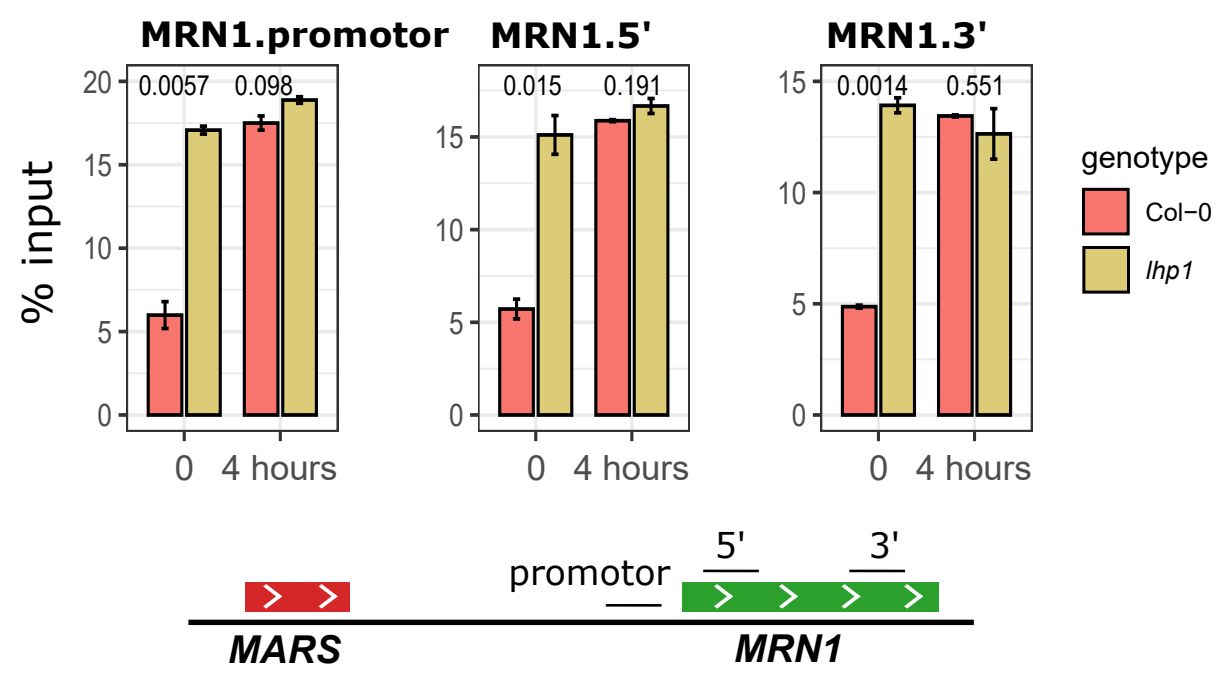

\section{LHP1 RIP}

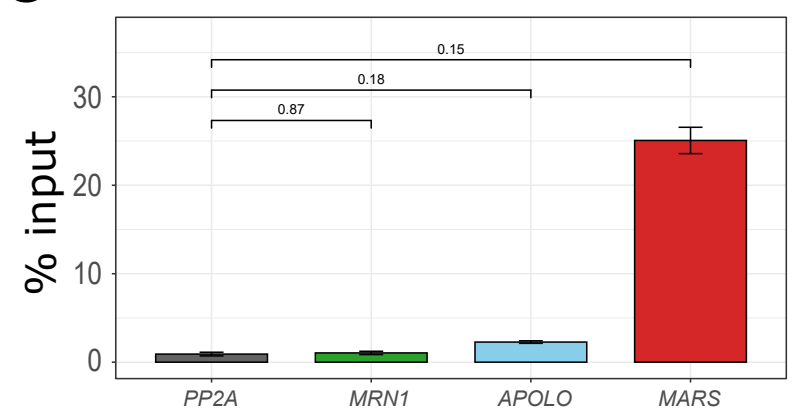

d LHP1 ChIP, MRN1.promoter

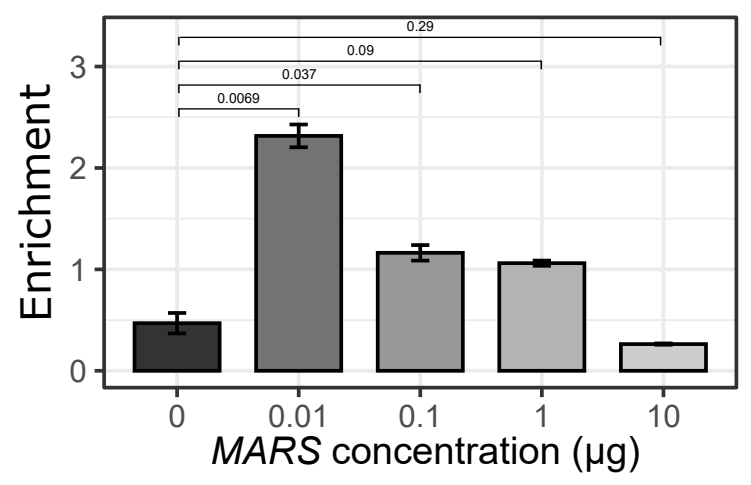

Fig. 4, MARS influences chromatin condensation of MRN1 gene through its interaction with LHP1 protein

a, Chromatin condensation in MRN1 gene of Col-0 and RNAi-MARS seedlings in control conditions and in response to ABA, determined by Formaldehyde Assisted Isolation of Regulatory Element (FAIRE)-qPCR.

b, Evolution of the chromatin condensation in MRN1 gene of Col-0 and Ihp1 mutant subjected to ABA treatment determined by Formaldehyde Assisted Isolation of Regulatory Element (FAIRE) qPCR.

In $\mathbf{a}$ and $\mathbf{b}$, results are expressed as the mean \pm standard error $(n=3)$ of the percentage of input (signal measured before isolation of decondensed region of chromatin, free of nucleosomes). Lower value indicates more condensed chromatin. Numbers are $p$-value of the difference between the two genotypes determined by Student ttest.

c, LHP1-MARS interaction was assessed by RNA immunoprecipitation (RIP) using LHP1GFP seedlings. Negative controls include a housekeeping gene (PP2A) and MRN1 mRNA. The interaction between $A P O L O$ and LHP1 was taken as a positive control ${ }^{15}$. Results are expressed as the mean \pm standard error $(n=4)$ of the percentage of input (signal measured before immunoprecipitation).

d, LHP1 binding to the MRN1 promoter region in chromatin from RNAi-MARS seedlings upon increasing amounts of in-vitro transcribed MARS RNA. After incubation (see Methods), the samples were crosslinked for LHP1 ChIP-qPCR. Higher values indicate more LHP1-DNA interaction. Results are expressed as the mean \pm standard error $(n=2)$ of the LHP1/lgg ratio. 


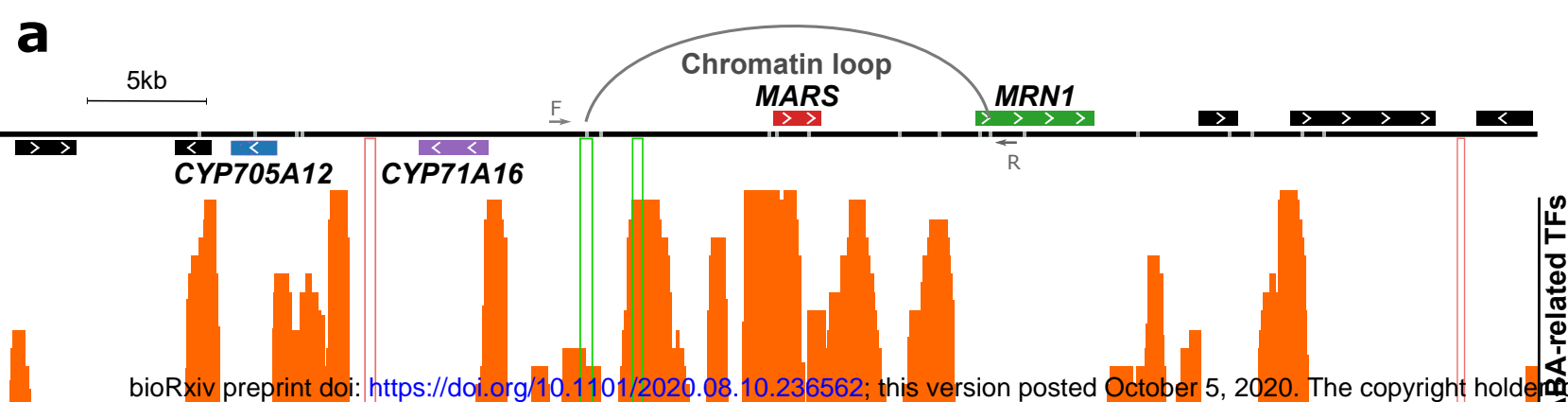

bioRxiv preprint doi: https://doi.org/10.1101/2020.08.10.236562; this version posted October 5, 2020. The copyright holderm
(which was not certified by peer review) is the author/funder. All rights reserved. No reuse allowed without permis

C. 1212

C.2

Negative Control Intergenic portion Negative Control

b

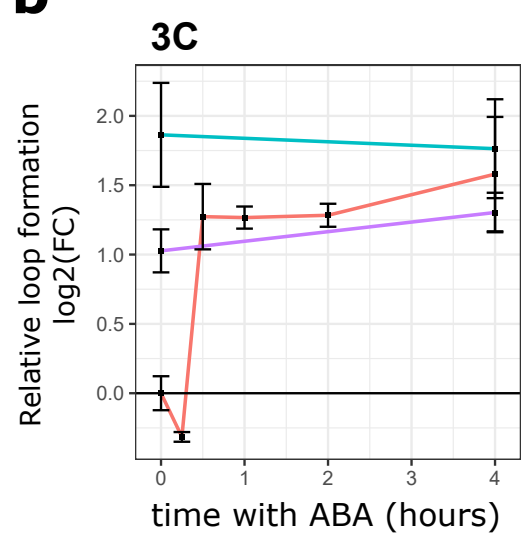

genotypes

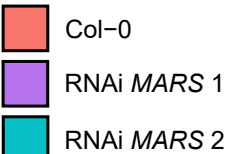

time with $A B A$ (hours)
C

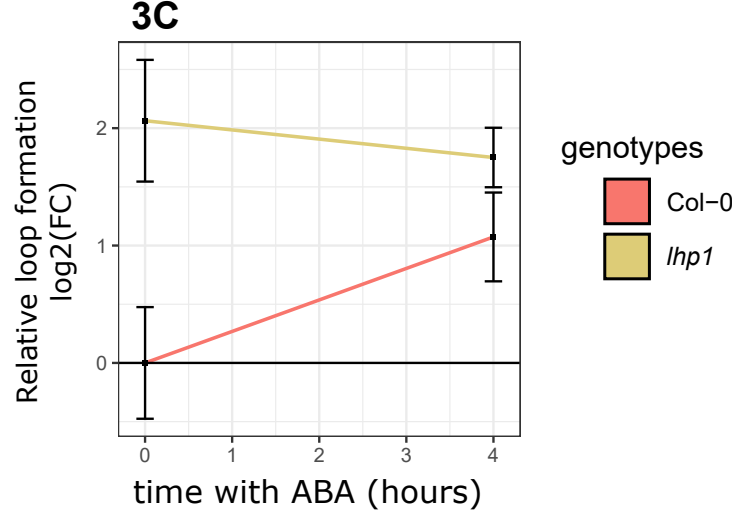

\begin{tabular}{|l|l|l|}
\hline $35 S$ sub-unit & mini35S & GUS \\
\hline
\end{tabular}

\begin{tabular}{|l|l|l|}
\hline Negative C.1 & mini35S & GUS \\
\hline
\end{tabular}

\begin{tabular}{|l|l|l|}
\hline Negative C.2 & mini35S & GUS \\
\hline
\end{tabular}

\begin{tabular}{l|l|l} 
Intergenic 1 & mini35s & GUS \\
\hline
\end{tabular}

\begin{tabular}{|l|l|l|}
\hline Intergenic 2 & mini35s & GUS \\
\hline
\end{tabular}
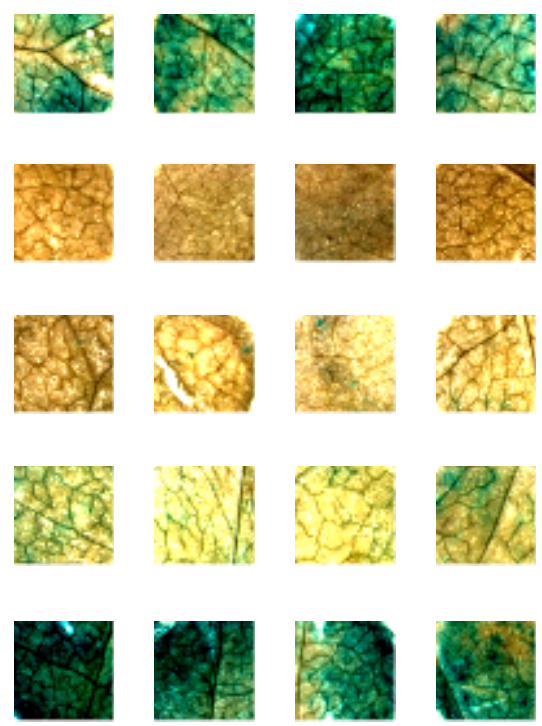

Fig. 5, An LHP1-dependent chromatin loop brings together the MRN1 locus and a putative enhancer element in response to $A B A$

a, Schematic illustration of the loop linking the MRN1 locus with the intergenic region between CYP71A16 and MARS. Forward $(F)$ and Reverse $(R)$ oligonucleotides used for 3CqPCR (in $\mathrm{B}-\mathrm{C}$ ) are indicated with arrows. The orange track shows the number of different ABA-related transcription factor binding sites (HB6, HB7, GBF2, GBF3, MYB3, MYB44, NFYC2, NF-YB2, ANAC102, ANAC032, ABF1, ABF3, ABF4, RD26, ZAT6, FBH3, DREBA2A, AT5G04760, HAT22 and HSFA6A) found along the marneral cluster ${ }^{30}$. Green and red rectangles indicate the putative enhancer region and the negative controls, respectively, tested for the GUS-based reporter system in $\mathbf{d}$.

b, Relative chromatin loop formation in response to ABA in Col-0 and RNAi-MARS seedlings. Results are expressed as the mean \pm standard error $(n=2)$ from $3 C$-qPCR using primer $F$ and $\mathrm{R}$ shown on a, compared to time $\mathrm{Oh}$.

c, Relative chromatin loop formation in response to ABA treatment in Col-0 and Ihp1 mutant. Data are represented as the mean \pm standard error $(n=3)$ from 3C-qPCR using primer $F$ and $\mathrm{R}$ shown on a, compared to time $\mathrm{Oh}$.

d, Constructs used for GUS-based reporter system are illustrated on the left. Corresponding transformed tobacco leaf discs are on the right $(n=4)$. First line represents the positive control in which $35 \mathrm{~S}$ sub-unit controls GUS expression. The second and third lines show two independent negative controls in which GUS gene is driven by a genomic region that does not contain ABA-related binding sites as indicated in $\mathbf{a}$. In the remaining lines, the transcriptional activity is assessed for the two intergenic regions indicated in a. 

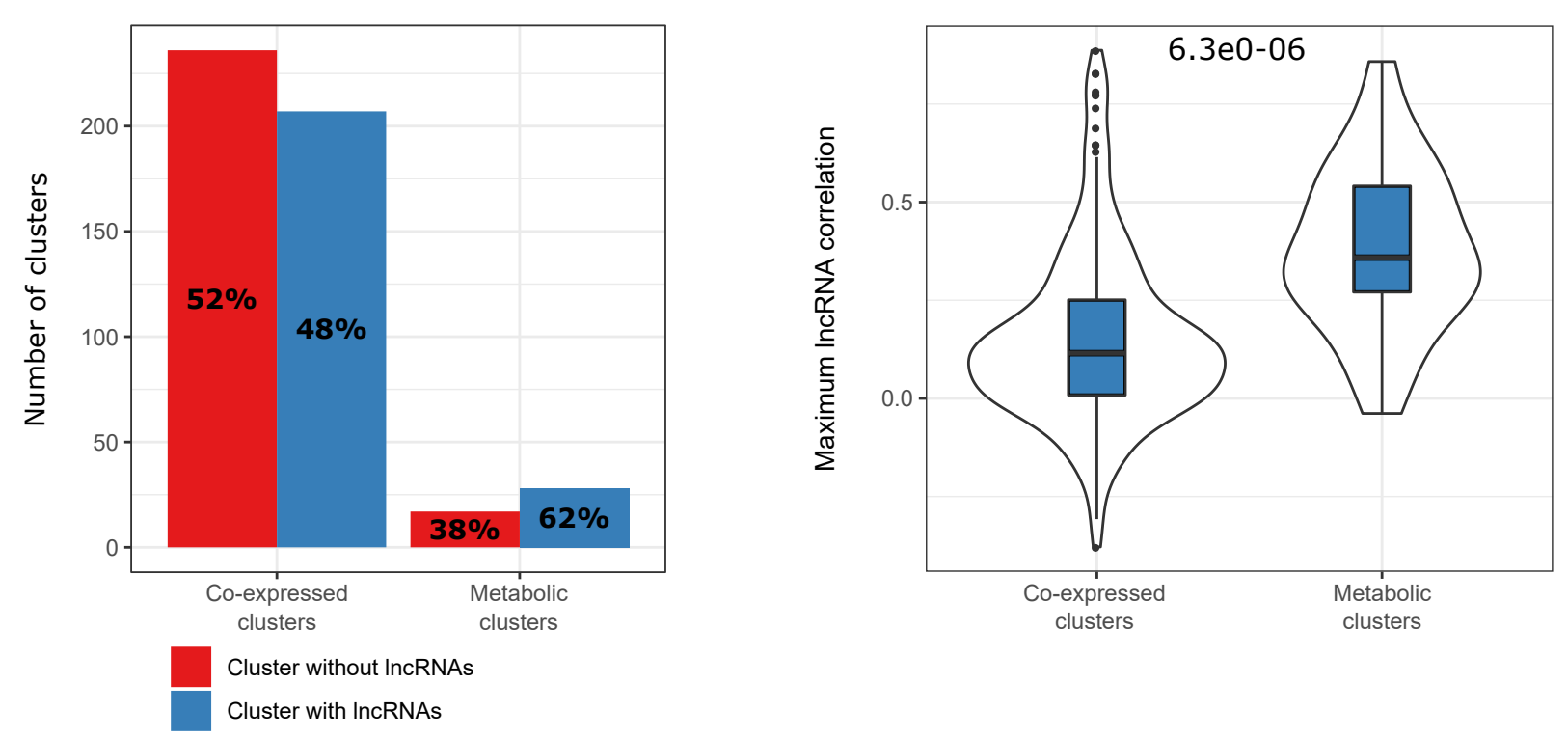

C

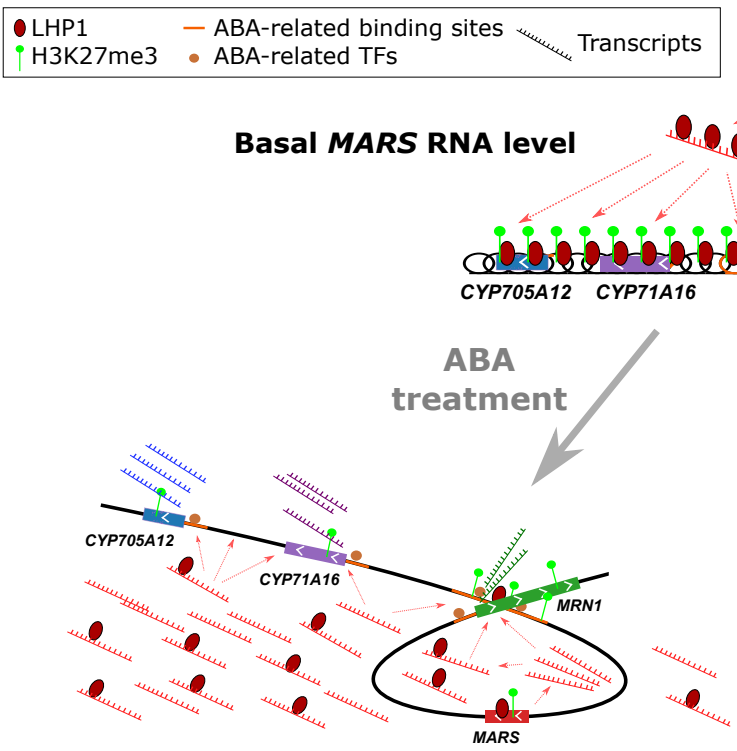

High MARS RNA level 